\title{
Overview of Biochemical Abnormalities and Molecular Genetics of Adenosine Deaminase Deficiency
}

\author{
ROCHELLE HIRSCHHORN \\ Department of Medicine, New York University Medical Center, New York, New York 10016
}

\begin{abstract}
Approximately 20 years ago, Giblett and coworkers serendipitously discovered that in some patients with the syndrome of severe combined immunodeficiency, the disease is due to an inherited deficiency of the enzyme adenosine deaminase (ADA). This then led to the discovery that inherited deficiency of the next enzyme in the same pathway for purine salvage, purine nucleoside phosphorylase, results in a profound defect in cell-mediated immunity. These two disorders, sometimes termed "purinergic immunodeficiency disorders," were the first of the inherited immunodeficiency disorders in which the specific molecular basis was determined. Although both are rare diseases, they are of importance for several reasons. First, they are among the few inherited disorders of which some children can be cured by a single treatment; second, they are ideally suited for gene therapy; and third, the pathologic mechanisms can tell us more about the nature of the immune system and have already allowed development of chemotherapy for some malignancies of the immune system. Knowledge of the specific defects has also facilitated diagnosis, counseling of families, and development of new approaches to therapy. This article focuses on ADA deficiency, briefly reviews the clinical and biochemical findings to provide a background for the next two articles in this supplement issue, and details work in progress with respect to several aspects of the specific molecular defects in different patients. Some of the generalizations that are beginning to emerge from studies of other disease loci as to the nature, diversity, and distribution of mutations at different genetic loci and the emerging correlations of phenotype with genotype are reviewed to provide a framework for examining the molecular defects in ADA deficiency. The status of our knowledge of the ADA locus with respect to analysis of mutations and possible correlations with genotype is then considered. It is important to note that although the ADA gene has been isolated and the gene product is known there are still unanswered questions. (Pediatr Res 33 (Suppl): S35-S41, 1993)
\end{abstract}

\section{Abbreviations}

ADA, adenosine deaminase

SAH, S-adenosyl homocysteine

SCID, severe combined immunodeficiency

\section{ADA DEFICIENCY AND SCID}

As reviewed by Dr. Puck in this supplement issue, SCID is classically an infantile onset syndrome with clinical manifesta-

Correspondence: Rochelle Hirschhorn, M.D., Department of Medicine, New York University Medical Center, 550 First Ave., New York, NY 10016. tions that result from a profound defect of both cellular and humoral immunity. If untreated, these clinical manifestations lead rapidly to death. The full-blown classic syndrome is one of overwhelming fungal, viral, and bacterial infections and failure to thrive, in a setting of lymphopenia and absence of nonmaternally derived $\mathrm{Ig}$. The syndrome may not become apparent until several weeks to months of life. The earliest studies of families with SCID revealed that there are several genetically different forms of the syndrome. In some families, the disease clearly shows recessive inheritance linked to the $\mathrm{X}$ chromosome, with only males affected, whereas in other families the inheritance is autosomal recessive, affecting both males and females. Based on several studies (reviewed in 1 and 2), it can be estimated that in approximately half of the children with autosomal recessive SCID (or approximately $20 \%$ of all SCID individuals, because of the greater incidence of the X-linked disorder) the disease is due to inherited deficiency of the purine salvage enzyme ADA (1-8).

Clinical spectrum. It is now apparent that the clinical phenotype of ADA deficiency encompasses a spectrum of disease including classic, fulminant neonatal onset SCID; a slightly delayed onset SCID accompanied by retention of some humoral immunity; a much later onset, progressive disease; and a "partial" ADA deficiency with apparently normal immune function (Table 1) (9-12).

The majority of infants initially described with SCID due to deficiency of ADA (classic ADA-SCID) were not grossly different clinically or immunologically from patients with SCID due to other as yet undefined causes. Although the major pathology and clinical manifestations are secondary to destruction of the immune system, involvement of other organs can be seen in ADASCID. Thus, half of ADA-SCID individuals have bony abnormalities, most notably cupping and flaring of the costochondral junctions, which are pathognomonic histologically and highly suggestive of the diagnosis on radiologic examination. Unusual renal and adrenal lesions as well as neurologic abnormalities have also been found at autopsy and may also be pathognomonic (reviewed in 13). Even initially, it was apparent that approximately $10 \%$ of ADA-SCID patients had a slightly delayed onset of disease and an initially milder course with retention of B lymphoid cells and some humoral immunity. Some of this variation occurred within families and therefore probably reflected environmental differences, such as extent of protection from infectious agents, rapidity of institution of antibiotic therapy, etc. In addition, as with other disorders, severity of disease may also be modified by effects of other interacting genetic loci that are unlinked and therefore segregate independently of the ADA mutation in a family (epistasis). With the application of testing for ADA deficiency to immunodeficient patients who do not present with classic SCID, even milder forms of immunodeficiency due to ADA deficiency have been found. In such patients, onset of clinically significant symptoms leading to diagnosis may be delayed until 3 to 5 y of age and there may be 
Table 1. Differing clinical phenotypes of ADA-deficient individuals

1. SCID (85-90\%): Indistinguishable from non-ADA-deficient SCID individuals (except for a bony lesion in half)

2. Delayed onset (10-15\%): Predominantly cellular immunodeficiency but specific antibody markedly deficient and Ig eventually absent

3. Late onset: Diagnosis not until 5-8 y of age; at time of diagnosis predominantly cellular immunodeficiency and some specific antibodies normal

4. "Partially" ADA deficient, ?immunologically normal: Ascertained by screening normal infants (newborns in New York State and African tribes)

marked predominance of abnormalities of cellular as compared with humoral immunity. Autoimmunity and eosinophilia may be relatively common in such children. In retrospect, many of these children would appear to have had increased numbers of infections before diagnosis, but the infections were not serious enough to lead to prolonged hospitalization. It will not be surprising if an "adult onset" form of the disease becomes apparent. At the extreme opposite end of the spectrum from SCID, several children with ADA deficiency in erythrocytes have been found who are grossly immunologically normal and currently between 4 and 15 y of age. These children (found as a result of screening newborns for ADA deficiency or screening populations for normal genetic variants of ADA and not because of disease) lack ADA in erythrocytes but have between 5 and $80 \%$ ADA activity in other cell types, presumably reflecting a mutant enzyme that is unstable primarily in erythrocytes. Such children have been termed "partial" ADA deficients (see 12 and references therein).

Biochemistry and metabolites. ADA is an enzyme that is present in all cells, yet its absence results in damage primarily to the immune system. Examination of the role of ADA in the normal individual has provided some clues to this apparent paradox. ADA is an enzyme of the purine salvage pathway that irreversibly removes an amino group from the naturally occurring purines, adenosine and deoxyadenosine, generating inosine and deoxyinosine. These latter compounds are either "salvaged" back to adenosine or other purines for reuse or further metabolized to uric acid and excreted. Adenosine is a component of RNA and the important and abundant energy-holding compound ATP, and deoxyadenosine is a major component of DNA. Deoxyadenosine is derived primarily from breakdown of cells with a rapid turnover, such as gut, epithelium, and, perhaps most significantly, from the extensive cell death of maturing lymphocytes within the thymus. Deoxyadenosine can then either be taken up by neighboring viable cells via active transport sites and be deaminated or escape into the plasma where it is taken up via active transport sites by other cell types and normally deaminated. Adenosine is derived similarly from dying cells and also is derived from the breakdown and recycling of ATP. When adenosine and deoxyadenosine (as well as several naturally occurring methylated adenosine compounds) cannot be degraded because of deficiency of ADA, these compounds accumulate in the plasma, are taken up by transport sites into cells, or, particularly for deoxyadenosine, are excreted in massive amounts. Patients with ADA deficiency have increased amounts of the substrates adenosine and deoxyadenosine in their plasma and excrete enormous amounts of deoxyadenosine in their urine (Table 2). In addition, because of the absence of the normal deamination of deoxyadenosine to deoxyinosine, the deoxyadenosine within cells is "pushed" along a normally minor pathway and is phosphorylated to deoxyATP, which is then trapped within cells and is no longer directly in equilibrium with the extracellular fluid. As a result, deoxyATP accumulates in enormous concentrations within cells, most easily demonstrable in the red cell. Last, a secondary inactivation of the enzyme SAH hydrolase has been demonstrated in erythrocytes of affected children (see below for discussion) (see 3, 14-17, and references therein).
Table 2. Biochemical abnormalities in ADA deficiency associated with immunodeficiency

1. Absence of ADA activity in erythrocytes, lymphocytes, and all tissues examined

2. Accumulation of deoxyATP in cells (erythrocytes, mononuclear cells, platelets, and kidney) (>200-fold increase)

3. Increased concentrations of adenosine and deoxyadenosine in plasma (adenosine > deoxyadenosine)

4. Excretion of deoxyadenosine, adenosine, and methylated adenosine derivatives in urine (deoxyadenosine $\gg \gg$ adenosine)

5. Secondary inhibition of SAH hydrolase

Table 3. Proposed mechanisms of toxicity in ADA deficiency

Metabolite In vitro effects

DeoxyATP Inhibits ribonucleotide reductase (required for normal DNA synthesis)

Deoxyadenosine Chromosome breakage

Inactivation of SAH hydrolase (inhibition of methylation reactions)

Adenosine

Multiple effects at adenosine receptors (probably least clinically significant)

Pathophysiology. There are several proposed mechanisms whereby the absence of ADA results in toxicity to cells of the immune system (Table 3). The first of these involves the accumulation of deoxyATP. It has been known for some time that deoxyATP is a very potent inhibitor of the enzyme ribonucleotide reductase and that this enzyme is required for normal DNA synthesis. Therefore, it has been proposed that there are certain tissues, most notably of the T-dependent immune system, in which there is preferential phosphorylation of deoxyadenosine to deoxyATP with a relative lack of dephosphorylation. In vitro evidence has been provided to support this proposal. In such cell types, there would be an increased steady state concentration of deoxyATP with subsequent inhibition of DNA synthesis. It has also been proposed, again on the basis of in vitro experiments, that deoxyadenosine directly results in chromosome breakage. It has also been proposed and demonstrated both in vivo and in vitro that there is secondary inactivation of the enzyme $\mathrm{SAH}$ hydrolase. Such inactivation would theoretically result in inhibition of methylation reactions in the body. Indeed, the very marked secondary inhibition of SAH hydrolase in red cells from these children provides perhaps the most sensitive measure of correction of metabolite concentrations after therapeutic maneuvers. Last, adenosine has multiple effects at adenosine A1 and A2 receptors, although none of these effects has been shown to be clinically significant. However, the marked lethargy and inability to follow visually, seen in some patients, may be contributed to by the known effects of adenosine at its receptors within the brain. It would appear reasonable that each of these several mechanisms may be operative but in different types of lymphoid cells and at different stages of differentiation. For example, maturing thymocytes might be exposed to unusually high concentrations of deoxyadenosine because of the extensive cell death that accompanies maturation to immunocompetent lymphocytes in the thymus.

An alternative argument has been proposed to explain the profound damage to the immune system. This argument is based upon the observation that, although ADA is a ubiquitous "housekeeping" enzyme, the activity of the enzyme varies over a 100fold range if erythrocyte activity is included and over a 10 -fold range if all nonerythroid tissues are examined. Teleologically, because cells of the immune system exhibit very high ADA activity, they would be the cells most profoundly affected by deficiency of the enzyme. This argument does not explain the lack of major involvement of other tissues that exhibit high ADA activity. Clearly, there are unanswered questions as to the patho- 
physiologic mechanisms. This is perhaps underscored by the observations that administration of an inhibitor of ADA (deoxycoformycin) has been effective in treating types of lymphoid malignancies that were not predicted by either the initial in vitro studies or by observations in ADA-SCID (see 3 for references therein).

Correlation of phenotype, in vitro enzyme activity and concentrations of metabolites. It would appear possible to inversely correlate residual ADA activity with severity of disease. Very clearly, partially ADA-deficient, immunologically normal children have greater residual ADA activity in their lymphoid cells than do ADA-SCID children. The lowest activity found in such children is approximately $5 \%$ of normal (12). At least one child with delayed onset disease has 1 to $2 \%$ of normal activity in lymphoid cells, which can be shown to be due to activity at the ADA locus (unpublished data). Therefore, the amount of residual ADA activity needed for normal immunologic function would appear to be between 1 and $5 \%$ of normal. We have recently been able to detect residual albeit trace amounts of ADA activity in some, but not all, patients by expressing recombinant mutant cDNA in reporter cells (COS) (unpublished data) (see below for more detailed discussion). In contrast, using standard assays, the residual ADA activity in patients with classic SCID is essentially not detectable (18). It should be noted that such standard in vitro assays of enzyme activity are made under artificial conditions not applicable to in vivo conditions, and they are unlikely to accurately reflect "whole body" functional ADA activity in all circumstances.

There is a gross correlation between the severity of the immune dysfunction and either the concentration of deoxyATP or excretion of deoxyadenosine. Thus, "partially" ADA-deficient, immunologically normal children have slightly increased concentrations of deoxyATP and excrete detectable deoxyadenosine in their urine. Classic ADA-SCID patients have a 100 - to 300 -fold increased concentration of deoxyATP, equaling or exceeding the concentration of ATP. These children similarly excrete massive amounts of deoxyadenosine in urine. Children with late onset immunodeficiency accumulate concentrations of deoxyATP intermediate between the SCID patients and the "partially" deficient patients. The excretion of deoxyadenosine is similarly intermediate. The concentrations of metabolites thus appear to provide a gross "whole body" in vivo assay of the residual ADA activity in these children and could possibly provide the best discriminant as to differences between patients.

Prenatal diagnosis. Prenatal diagnosis is possible using either chorionic villous biopsy or amniotic cells and determination of enzyme activity. DNA-based diagnosis is also theoretically possible using normal variants at the ADA locus or by direct detection of mutations (see below). Using DNA-based methods, preimplantation diagnosis should also be feasible eventually (19 and references therein).

Therapy. At the present time, there are four modes of therapy available (Table 4). When a related matched donor is available, histocompatible bone marrow transplantation is the therapy of choice, with appearance of normal immune function as well as almost complete reduction in concentrations of metabolites. In some patients, there is engraftment only of T cells and not of B cells but with apparent function of the ADA-deficient B cells. Unfortunately, because of average family size in the Western world, less than one third of children will have such a matched donor. Haploidentical bone marrow transplantation has been used, but there has been much debate as to whether ADAdeficient patients respond as well to haploidentical transplanta-

Table 4. Therapy of $A D A$ deficiency

1. Histocompatible bone marrow transplant (current treatment of choice)
2. Haploidentical bone marrow transplant
3. Enzyme replacement
4. Gene replacement

tion and whether they require more marrow ablation before transplantation than do patients with other forms of SCID. Nonetheless, there are patients with ADA deficiency who have had successful transplantations of haploidentical marrow without any prior ablation. Enzyme replacement is a third approach. Initially, an incomplete form of enzyme replacement was provided by partial exchange transfusions. This therapy was of limited efficacy in a few patients and had no discernible effect in most patients. More recently, partial exchange transfusion has been replaced by the more efficient and safer direct administration of larger amounts of a modified form of ADA, discussed in detail by Dr. Hershfield in this supplement issue. The fourth approach, gene replacement therapy of T cells, is addressed by Dr. Blaese (this issue). I would like to raise the speculation that the underlying genotype may play a role in modulating the outcome in all these forms of therapy (20-25).

\section{MUTATIONS AT ADA LOCUS}

Before examining the nature of mutations at the ADA locus (Table 5), it is worthwhile to consider the information available as to the nature of diseases causing mutations at other disease loci. Mutations that result in disease can be of several types. They include deletions (ranging from very large to single nucleotides); missense mutations (single basepair changes resulting in an amino acid substitution and either a nonfunctional or an unstable protein); nonsense (single basepair changes that introduce a stop codon and prevent translation of a complete protein and/or lead to unstable mRNA); and splicing mutations (basepair changes, usually single, that alter the normal splicing and result in abnormal mRNA). At some loci, such as that for Duchenne's muscular dystrophy and $\alpha$-globin, where there is either duplication of a similar sequence (Duchenne's muscular dystrophy) or duplication of loci ( $\alpha$-globin), there is a preponderance of large deletions, presumably resulting from mispairing of similar stretches of DNA (homologous recombination). At other loci, such as the $\beta$-globin locus, missense mutations predominate in phenotypes that result from altered function (altered affinity for oxygen) or physical properties (sickle cell mutation) of the $\beta$-globin chain. Mutations at the $\beta$-globin locus that result in virtual absence of the protein (thalassemias) are predominantly due to splicing mutations. Thus, the type of mutation may vary at different loci or with differing phenotypes at the same locus. It was also expected that there would be multiple different mutations at any given disease locus. However, it is becoming

Table 5. Mutations at ADA locus

\begin{tabular}{llll}
\hline Exon no. & $\begin{array}{c}\text { Basepair no. } \\
\text { (phenotype) }\end{array}$ & $\begin{array}{c}\text { Basepair no. } \\
\text { (change) }\end{array}$ & Amino acid \\
\hline Exon 1 & & & $\begin{array}{l}\text { Deletion } \\
\text { Exon 4 }\end{array}$ IVS 3 (SCID) \\
& & AG-GG & $\begin{array}{l}\text { Exon } \\
\text { spliced out } \\
\text { (out of frame) }\end{array}$
\end{tabular}

3' splice site

226 (partial)

301 (SCID)

302 (SCID)

320 (SCID)

Exon $5 \quad 446$ (partial)

466 (SCID)

Exon $7 \quad 631$ (partial)

632 (SCID)

643 (partial) 646 (SCID)

Exon $9 \quad 821$ (partial)

Exon 10

872 (SCID)

890 (partial)

911 (SCID)

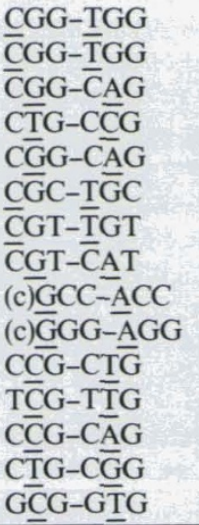

Arg76Trp

Arg 101Trp

Arg101Gln

Leu 107Pro

Arg149Gln

Arg156Cys

Arg211Cys

Arg211His

Ala215Thr

Gly216Arg

Pro274Leu

Ser291Leu

Pro297Gln

Leu304Arg

Exon 11

Ala329Val 
apparent that at several disease loci a limited number of mutations may be responsible for disease in the majority of patients. This predominance of a limited number of mutations could occur through several mechanisms including selective advantage of the mutation in asymptomatic carriers, inbreeding, founder effect, and recurrence at "hot spots" for mutation. In a particular ethnic group, only a few mutations may account for the disease in over $90 \%$ of patients. Recurrent mutations in unrelated individuals of very different ethnic origins may be due to mutations at hot spots. One type of hot spot appears to be at $\mathrm{CpG}$ dinucleotides. It has been known for some time that cytosine, if methylated to 5-methylcytosine, will spontaneously deaminate to thymine. In man and mammals, as opposed to bacteria, cytosine is usually methylated only when it is followed by guanosine or at $\mathrm{CpG}$ dinucleotides. If a methylated cytosine on the sense or coding strand of the double-stranded DNA is deaminated to thymine, a $\mathrm{C}$ to $\mathrm{T}$ transition will occur. If the deamination occurs initially on the antisense strand, copying of the mutated strand will result in a $G$ to $A$ transition on the sense strand. Thus, $C$ to $\mathrm{T}$ and $\mathrm{G}$ to $\mathrm{A}$ transitions at $\mathrm{CpG}$ dinucleotides appear to represent hot spot mutations with a likelihood of recurring independently and being found in multiple unrelated individuals.

In ADA deficiency of all clinical phenotypes, the majority of mutations are changes of a single nucleotide that result in change of a single amino acid (missense mutations). Such mutations result in either unstable or inactive protein. Fifteen different missense mutations have been reported at the ADA locus, nine in ADA-SCID patients and six in partially deficient individuals (Table 5). A single splicing mutation has also been reported (2633). The noncoding regions (introns) of the ADA structural gene are very rich in a form of repetitive DNA termed "Alu repeats," providing a substrate for deletions by homologous recombination. However, only a single deletion resulting from homologous recombination at Alu repeats has been reported to date in an ADA-deficient SCID patient. The deletion abolishes the promoter region as well as exon 1 and has been reported in two apparently unrelated SCID patients homozygous for the same deletion $(34,35)$. We have recently characterized an additional large deletion, also including the promoter region, that does not involve homologous recombination. The homozygous occurrence of a deletion that by definition completely abolishes ADA activity is of special significance because this indicates that complete absence of the ADA enzyme appears to be compatible with life. (However, the reproductive history with respect to fertility and miscarriages in such families has not been reported and would be of interest, inasmuch as it has been shown very convincingly in murine species that ADA may be very important for normal development in utero.) Twelve of these 15 missense mutations are hot spot mutations or $\mathrm{C}$ to $\mathrm{T}$ or $\mathrm{G}$ to $\mathrm{A}$ transitions at $\mathrm{CpG}$ dinucleotides and therefore are candidates for independent recurrence in unrelated patients. This $75 \%$ frequency is much higher than the approximately $30 \%$ frequency found in a survey of mutations at multiple other loci for genetic disease (36).

In partially ADA-deficient patients, it was not unexpected that the same mutation would be found in more than one individual, inasmuch as there was a common geographic and/or racial origin of many of these patients consistent with a common ancestor (Table 6). The first of these children was ascertained during population surveys to determine the frequency of the gene coding for a normal biochemical variant of ADA among the Kung tribesmen in the Kalahari desert of Africa. Similar to subsequent children, although he lacked ADA activity in his erythrocytes, he was found to have enzyme in his lymphoid cells (37). Shortly after that, New York State instituted a newborn screening program for detection of ADA-deficient SCID. Although the test used failed to ascertain at least two siblings who had ADA-SCID (unpublished data), it did detect a group of children who were partially ADA deficient. Of note, all but one of the children either came from a limited area in the Caribbean or were of African-American descent, a frequency out of proportion to that
Table 6. Frequency of mutations and biochemical phenotype in seven partially ADA-deficient individuals

\begin{tabular}{lccc}
\hline Mutation & $\begin{array}{c}\text { No. of } \\
\text { chromosomes }\end{array}$ & $\begin{array}{c}\text { No. of } \\
\text { children }\end{array}$ & $\begin{array}{c}\text { Activity }(\% \\
\text { normal) }\end{array}$ \\
\hline Arg76Trp & 4 & 3 & 16 \\
Leu107Pro* & 2 & 2 & 0 \\
Arg149Gln & 1 & 1 & 42 \\
Arg211Cys & 1 & 1 & 8 \\
Ala215Thr & 2 & 1 & 8 \\
Pro274Leu & 1 & 1 & 12 \\
Pro297Gln & 3 & 2 & 28 \\
\hline
\end{tabular}

* Also found in ADA-SCID; in partially ADA-deficient patients, found together with (heteroallelic) Arg211Cys or Arg76Trp.

Table 7. Frequency of six missense mutations in 45 ADA-SCID chromosomes

\begin{tabular}{lcl}
\hline \multicolumn{1}{c}{ Mutation } & $\begin{array}{c}\text { No. of } \\
\text { chromosomes }\end{array}$ & \multicolumn{1}{c}{ ADA-SCID patient } \\
\hline Leu107Pro & 2 & GM3136; Fa \\
Arg211His & 2 & GM2756; GM2606 \\
Gly216Arg & $4^{*}$ & GM1390; GM2436; GM11411* \\
Ser291Leu & 1 & GM2471 \\
Leu304Arg & 1 & GM2471 \\
Ala329Val & $6^{*}$ & GM2756; GM2825; GM4258; DM*; Te \\
Total & $16 / 45$ & \\
\hline
\end{tabular}

* Indicates patients who are homozygous for the mutation. All other patients were heterozygous for mutations.

of these groups in the population screened. When we determined the mutations in seven of these children, we did indeed find the same mutation in several children $(32,33$, and references therein) (Table 6). However, there were also seven different mutations and most of the children carried two different mutations. There are several possible explanations for the occurrence of multiple mutations at high frequency in a limited population. Because diagnosis requires two deficiency alleles, the occurrence of one or two frequent mutations in the population (because of founder effect, inbreeding, and/or genetic drift) would also lead to the ascertainment of very rare mutations. Alternatively, there could be a selective advantage for carriers of one partial deficiency mutation in the area from which this population derived, as there is for the $\mathrm{Hb}$ sickle mutation. We have also been able to correlate the molecular genotype with the biochemical phenotype and determine the relative enzyme activity of each of the mutations (as well as other alterations in biochemical properties of the mutant enzymes) (see below for more detailed discussion). Of note, one of the mutations found in these children results in loss of enzyme activity in all cell types and was subsequently found in ADA-SCID patients.

In a very rare autosomal recessive disorder such as ADAdeficient SCID $(<1 / 500000)$, one would expect to find, in an outbred population, an increased frequency of consanguinity with homozygosity in such families but only rare occurrence of the same mutation in unrelated individuals. Unexpectedly, it is clear that several mutations are relatively common in ADA deficiency with SCID. Thus, we have surveyed 45 ADA-SCID chromosomes from 23 unrelated children for the presence of six mutations that we determined could be detected by polymerase chain reaction amplification and digestion with restriction enzymes $(31,38)$ (Table 7$)$. These six mutations accounted for more than one third of the mutant alleles studied (16 of 45) and were found in 14 children. Most of the patients were heteroallelic, carrying two different mutations on each of their chromosome 20s. Two patients, both from inbred communities, were homozygous, each for a different mutation, reducing the maximum number of independent chromosomes surveyed from 45 to 43. Four of the mutations (Ala329Val, Gly216Arg, Leu 107Pro, and Arg211His) were found in more than one unrelated individual. 
The Ala329Val mutation would appear to have a high frequency among patients of African-American background and also would occur in Caucasians on a different chromosomal background. Markert et al. (39) have also reported that this mutation is relatively common, although the frequency is overestimated by combining the two studies [Hirschhorn et al. (38) and Markert et al. (39)] because some of the patients are duplicated. The Ala329Val mutation is at a CG dinucleotide and has been shown to have recurred independently. The second most frequent mutation, Gly216Arg, was initially detected in an Amish child and also in a Swiss child from the area of Switzerland from which the Amish originated. The occurrence of this Gly216Arg mutation in these two children could reflect a common ancestor. The Gly216Arg mutation was also found in a third child of undefined ancestry; because the mutation is also a hot spot mutation, it could have resulted from independent recurrence of the mutation. Two additional mutations (Leu107Pro and Arg211His), both of which are $\mathrm{CpG}$ hot spot mutations, were each found in two unrelated children. Last, two mutations (Arg156Cys and Leu304Arg) were each unique to the same patient. We have also identified, either definitively or tentatively (by abnormal restriction fragments), seven additional mutations, including a deletion. One of these mutations may be relatively common, whereas a second was found in homozygosity in a child from Newfoundland. In an autosomal recessive disorder, one expects to find increased consanguinity and increased homozygosity. Three children were homozygous for mutations, and all three were from groups with high inbreeding, such as the Amish, Louisiana blacks, and Newfoundlanders. Therefore, there is evidence that the occurrence of the same mutation at the ADA locus in apparently unrelated children results from both founder effect and by independent recurrence at mutational hot spots.

\section{CORRELATION OF GENOTYPE AND PHENOTYPE IN ADA DEFICIENCY}

Phenotype/genotype correlations have now been made in a number of inherited disorders, as discussed above, and the list gets longer every day. Thus, a specific mutation in homozygosity can give rise to a severely affected phenotype, and a different homozygous mutation at a different site in the same gene can give rise to milder or even adult onset phenotypes. At the ADA locus, we have yet to define an adult onset phenotype. In some cases, the phenotype can be clinically very different for mutations at different regions of the gene (osteogenesis imperfecta versus Ehlers-Danlos, both due to mutations in the collagen type I genes). Depending on the nature of the gene product, mutations that in homozygosity result in a relatively mild phenotype can in heterozygosity be either dominant or recessive to a "severe" mutation. At the ADA locus, the gene product is a monomeric enzyme and one would therefore expect that a "mild" mutation resulting in residual ADA activity would be dominant over a "severe" mutation in defining phenotype. Although phenotype/ genotype correlations may hold for the majority of patients with a particular disorder, the phenotype can be modified by environment or by other linked or unlinked modifying genes.

Is there a correlation between the genotype and the phenotype for ADA deficiency? We can correlate the mutations in partially ADA-deficient individuals with the normal clinical phenotype and with the biochemical phenotype. It would seem possible that some of these partial mutations, particularly the Arg211Cys and the Ala215Thr mutations that code for the lowest enzyme activity, could in combination with a total deficiency allele give rise to a late onset immunodeficiency. However, the finding in a partially ADA-deficient, immunologically normal child of a total deficiency allele (Leu 107Pro) in combination with the Arg211Cys mutation would be consistent with a mild mutation being dominant over the severe mutation in determining phenotype. With ADA-deficient SCID, correlations of genotype with phenotype will be more difficult. Because the disease is rare and most patients will carry two different mutations, there may be an insufficient number of patients who are homozygous for the same mutation to critically define phenotype/genotype correlations. In addition, it is not clear what parameters will most clearly define aspects of the phenotype that will correlate with genotype. We can define the phenotype clinically with respect to age of onset and/or immunologic abnormalities. In some children, the cellular immune dysfunction, as measured by in vitro tests, has been shown to be apparent in utero (reviewed in 19) and with onset of disease in the first few weeks of life. However, environment clearly may play a major role in the initial phenotype, independent of the underlying genotype, because the time of onset of disease and of aspects of the immune deficit can be modified by random variation in the actual time and nature of infectious agents that are met. Therefore, it may be difficult to classify patients by clinical phenotype because of a strong effect of environment. The phenotype could potentially also be defined by the amount of residual ADA activity, the extent of accumulation of toxic metabolites, or, most significantly, the response to various therapeutic modalities. Thus, haploidentical transplantation without prior ablation of marrow might be more frequently successful in patients with severe disease and a genotype such as a deletion, in which there is no possible residual ADA. In contrast, enzyme replacement might result in more complete reconstitution with lower doses and/or frequency of enzyme replacement in patients with some residual enzyme activity.

Despite these expected difficulties in establishing phenotype/ genotype correlation for ADA deficiency, several of the mutations that have been described are candidates for markers of differing clinical phenotype. For example, one of the patients examined from the Amish community, who presented with very fulminant, early onset disease and accumulation of extremely high concentrations of toxic metabolites, was homozygous for the Arg216Gly mutation. In a second patient, a mutation at the next codon (in this case in combination with a deletion) was associated with a similar, very severely affected phenotype (unpublished data). On the basis of the recent crystallographic analysis of murine ADA and comparison of sequence in different species $(40,41)$, it is apparent that these codons are in the region of the substrate binding site and also in an area conserved through evolution from Escherichia coli to man. Therefore, major alterations in the charge of the amino acids in this region should be very deleterious. These combined observations suggest that the Arg216Gly mutation in homozygosity or with a similar "severe" mutation may be associated with a severely affected phenotype. I would hypothesize, and I stress that this is a hypothesis, that these two particular mutations, in homozygosity or in combination with either another severe missense allele or a deletion, are candidates for markers of severe disease. Clearly, a heterozygous combination with a "mild" allele would be predicted to ameliorate the disease manifestations, with the effects of the mild allele being dominant. Conversely, we have recently identified a mutation that in homozygosity is associated with milder disease and retention of some residual ADA and would therefore be a candidate for a marker for milder disease. We have also recently demonstrated residual ADA activity for several other mutations found in the small number of children who exhibited the best responses to partial exchange transfusion (see below).

Another criterion for slightly less severe disease may have been provided in the form of incomplete, crude "enzyme replacement" provided by partial exchange transfusions, now replaced by the more effective direct enzyme replacement afforded by polyethylene glycol (PEG)-ADA. This crude replacement used multiple partial exchange transfusions, based on the rationale that red cells have both transport sites for taking up adenosine and deoxyadenosine from plasma and normal ADA for degrading these purines. The concentrations of deoxyATP in red cells and deoxyadenosine in urine can be dramatically decreased by partial exchange transfusion, albeit to a lesser extent than can be accom- 
plished with PEG-ADA. What also became apparent was that, in contrast to the dramatic response of the first patient reported, most patients did not show any clinical or immunologic response to partial exchange transfusion. However, six patients were reported in the literature as having responded to some degree, the response most clearly manifested by prolongation of life and, for several, the ability to attend school normally. Clearly, there was something unusual about these patients because two of them (and a sibling) survived to more than $10 \mathrm{y}$ of age, at which point they began receiving PEG-ADA. A third child is still alive as an adolescent without any therapy. The two remaining patients died, one at the age of 3 , ironically of autoimmune hemolytic anemia, and the other at 5, of chicken pox. (For the purposes of this analysis, it does not matter if the longevity reflected natural history of disease and/or the effect of the partial exchange transfusions.) These patients were included in the survey of 45 ADA - chromosomes discussed above.

We have now identified all but one of the mutations in this group of six "partially responsive" children (Table 8). Because the children are unrelated and there is no known consanguinity or derivation from a group with a high frequency of inbreeding, up to 12 different mutations might be expected. By contrast, mutations at only three codons account for over half of the mutant alleles (seven of 12). (Of note, three of the six children are of African-American origin.) The Ala329Val mutation accounted for three $(25 \%)$ of the alleles and, although this is a common mutation, it was found in only two other children among the 31 additional independent chromosomes that we examined (three of 12 versus two of 31). This difference in frequency is statistically significant. One of the patients carrying the Ala329Val mutation is a mosaic. One B lymphoid cell line carries the Ala329Val mutation on one allele, but expresses normal ADA from the second allele. A second cell line does not express mRNA from this second allele (unpublished data). Consistent with the hypothesis that the Ala329Val mutation may be associated with milder disease, we have preliminary evidence that the Ala329Val mutation codes for detectable enzyme activity. Two of the patients carry the Arg 211 His mutation and are the only two patients in the group of 43 independent chromosomes examined in which this mutation was found. Two additional patients each carried a mutation at codon 101 (Arg101Trp and Arg101Gln). Of note, the Arg101Trp mutation has been found to express catalytically active protein in T cells, consistent with a less severe mutation (42). The last patient to be discussed was the patient initially reported to have a dramatic response to red cell therapy (24). He carries two unique mutations not seen in any of the other children we surveyed, and therefore both the genotype and the phenotype with respect to response to therapy appear to be unusual. We have recently determined that both of his mutations code for detectable ADA activity. We would therefore suggest that several of the mutations found in these children may result in milder phenotypes. It remains to be determined whether this milder phenotype will be manifested by initial clinical and/or immunologic phenotype, response to replacement therapy, requirement for marrow ablation before haploidentical transplant, longevity, or only in the extent of residual

Table 8. Mutations in six ADA-SCID patients responsive* to enzyme replacement

\begin{tabular}{cll}
\hline & \multicolumn{2}{c}{ Mutation } \\
\cline { 2 - 3 } Patient & Chromosome 1 & Chromosome 2 \\
\hline GM2825 & Ala329Val & splice \\
GM2756 & Ala329Val & Arg211His \\
GM4258 & Ala329Val & Ser291Leu \\
GM2606 & Arg101Trp & Arg211His \\
GM1715 & Arg101Gln & $?$ \\
GM2471 & Arg156Cys & Leu304Arg \\
\hline
\end{tabular}

* See text for discussion. enzyme activity and/or accumulation of metabolites. We are currently determining the ability of additional mutations to code for residual enzyme activity.

Last, mutations in the currently smaller group of delayed and/ or late onset patients could identify mutations that would be more definitive markers for milder disease, particularly if present in homozygosity. However, in this group of patients, one would expect an even greater influence of environment on phenotype and therefore greater intrafamilial variation between individuals with the same genotype. We have identified a mutation in homozygosity in one such patient and found that there was expression of detectable ADA, albeit at approximately $1 \%$ of normal. The ADA was altered in charge, consistent with the nature of the mutation.

To summarize the current characteristics of mutations at the ADA locus, they are primarily missense mutations that appear to result in either unstable or inactive protein. Although this initially suggested that deletions might not be compatible with life, two children homozygous for the same deletion have been reported. There are many different mutations, but some are relatively common, which will greatly simplify attempts at DNAbased diagnosis. To explain the finding of common mutations in this rare autosomal recessive disorder, there is evidence for founder effect, inbreeding, common ancestors, and recurrence at the $\mathrm{CpG}$ hot spot. There is a spectrum of phenotypes, and at this point phenotype/genotype correlations are just emerging. ADA is a monomeric enzyme, and low levels of activity are sufficient for normal development. Because of this "threshold effect," mutations resulting in any degree of residual enzyme activity would be expected to result in lesser accumulations of toxic metabolites and a milder phenotype and to be dominant in defining a milder phenotype even if combined with a total deficiency mutant allele. Such correlations are going to be extremely difficult to establish for this particular disorder because of the marked effect of environment upon the phenotype and, in addition, the evidence that there may be some epistatic defects, namely interaction with other genetic loci. Nonetheless, given that this is one of the first disorders to be treated by both enzyme and gene replacement therapy, delineation of possible phenotype/genotype correlations may be significant for evaluating the outcome of such therapeutic maneuvers. Several mutations are candidates for markers of very severe disease and milder, delayed or late onset disease. I have attempted to be provocative in this presentation, but, clearly, additional studies will be required for a definitive evaluation of the molecular biology of ADA deficiency and its relation to clinical parameters.

\section{REFERENCES}

1. Hirschhorn R 1990 Adenosine deaminase deficiency. In: Rosen FS, Seligmann M (eds) Immunodeficiency Reviews, Vol 2. Harwood Academic Publications, London, pp 175-198

2. Hirschhorn R, Hirschhorn K 1990 Immunodeficiency disorders. In: Emery AEH, Rimoin DL (eds) The Principles and Practice of Medical Genetics. Churchill-Livingstone, Edinburgh, pp 1411-1430

3. Giblett ER, Anderson JE, Cohen F, Pollara B, Meuwissen HJ 1972 Adenosine deaminase deficiency in two patients with severely impaired cellular immunity. Lancet 2:1067-1069

4. Giblett ER, Ammann AJ, Wara DW, Sandman R, Diamond LK 1975 Nucleoside-phosphorylase deficiency in a child with severely defective T-cell immunity and normal B-cell immunity. Lancet 1:1010-1013

5. Kredich NM, Hershfield MS 1989 Immunodeficiency disease associated with adenosine deaminase deficiency and purine nucleoside phosphorylase deficiency. In: Scriver CM, Beaudet AL, Sly WS, Valle D (eds) The Metabolic Basis of Inherited Disease, 5th Ed. McGraw Hill, New York, pp 1045-1076

6. Hirschhorn R 1986 Inherited enzyme deficiencies and immunodeficiency: adenosine deaminase (ADA) and purine nucleoside phosphorylase (PNP) deficiencies. Clin Immunol Immunopathol 40:157-165

7. Hirschhorn R 1979 Incidence and prenatal detection of adenosine deaminase deficiency and purine nucleoside phosphorylase deficiency. In: Pollara B, Pickering RJ, Meuwissen HG, Porter I (eds) Inborn Errors of Specific Immunity. Academic Press, New York, pp 5-15

8. Hirschhorn R 1979 Clinical delineation of adenosine deaminase deficiency. In: Enzyme Defects and Immune Dysfunction, Ciba Foundation Symposium 68. Excerpta Medica, Amsterdam, pp 35-54

9. Levy Y, Hershfield MS, Fernandez-Mejia C, Polmar SH, Scudiery D, Berger 
M, Sorensen RU 1988 Adenosine deaminase deficiency with late onset of recurrent infections: response to treatment with polyethylene glycol-modified adenosine deaminase. J Pediatr 113:312-317

10. Morgan G, Levinsky RJ, Hugh-Jones K, Fairbanks LD, Morris GS, Simmonds HA 1987 Heterogeneity of biochemical, clinical and immunological parameters in severe combined immunodeficiency due to adenosine deaminase deficiency. Clin Exp Immunol 70:491-499

11. Geffner ME, Stiehm ER, Stephure D, Cowan MJ 1986 Probable autoimmune thyroid disease and combined immunodeficiency disease. Am J Dis Child 140:1194-1196

12. Hirschhorn R, Ellenbogen A 1986 Genetic heterogeneity in ADA deficiency: five different mutations in five new patients with partial ADA deficiency. Am J Hum Genet 38:13-25

13. Ratech H, Alba-Greco M, Gallo G, Rimoin DL, Kamino H, Hirschhorn R 1985 Pathologic findings in adenosine deaminase deficient-severe combined immunodeficiency I. Kidney, adrenal and chondro-osseous tissue alterations. Am J Pathol 120:157-169

14. Hirschhorn R, Ratech H, Rubinstein A, Papageorgiou P, Kesarwala H, Gelfand E, Roegner-Maniscalco V 1982 Increased excretion of modified adenine nucleosides by children with adenosine deaminase deficiency. Pediatr Res $16: 362-369$

15. Cohen A, Hirschhorn R, Horowitz SD, Rubinstein A, Polmar SH, Hong R, Martin Jr DW 1978 Deoxyadenosine triphosphate as a potentially toxic metabolite in adenosine deaminase deficiency. Proc Natl Acad Sci USA $75: 472-476$

16. Hirschhorn R, Roegner-Maniscalco V, Kuritsky L, Rosen FS 1981 Bone marrow transplantation only partially restores purine metabolites to norma in ADA deficient patients. J Clin Invest 68:1387-1393

17. Hershfield MS, Kredich NM 1979 In vivo inactivation of erythrocyte Sadenosylhomocysteine hydrolase by 2 '-deoxyadenosine in adenosine deaminase-deficient patients. J Clin Invest 63:807-811

18. Hirschhorn R, Roegner V, Jenkins T, Seaman C, Piomelli S, Borkowsky W 1979 Erythrocyte adenosine deaminase deficiency without immunodeficiency: evidence for an unstable mutant enzyme. J Clin Invest 64:11301139

19. Hirschhorn R 1986 Prenatal diagnosis of adenosine deaminase deficiency, purine nucleoside phosphorylase deficiency, and severe combined immunodeficiency of unknown etiology. In: Milunkski A (ed) Genetic Disorders and the Fetus: Diagnosis, Prevention, and Treatment, Vol 2. Plenum Press, New York, pp 419-424

20. Parkman R, Gelfand EW, Rosen FS, Sanderson A, Hirschhorn R 1975 Severe combined immunodeficiency and adenosine deaminase deficiency. N Engl J Med 292:714-719

21. Chen SH, Ochs HD, Scott CR 1978 Adenosine deaminase deficiency: disappearance of adenine deoxynucleotides from a patient's erythrocytes after successful marrow transplantation. J Clin Invest 62:1386-1389

22. Fischer A, Griscelli C, Friedrich W, Kubanek B, Levinsky R, Morgan G, Vossen J, Wagemaker G, Landais P 1986 Bone-marrow transplantation for immunodeficiencies and osteopetrosis: European Survey, 1968-1985. Lancet 2:1080-1084

23. Polmar SH, Stern RC, Schwartz AL, Wetzler EM, Chase PA, Hirschhorn R 1976 Enzyme replacement therapy for adenosine deaminase deficiency and severe combined immunodeficiency. N Engl J Med 295:1337-1343

24. Hershfield MS, Buckley RH, Greenberg ML, Melton AL, Schiff R, Hatem C, Kurtzberg J, Markert ML, Kobayashi RH, Kobayashi AL, Abuchowski A 1987 Treatment of adenosine deaminase deficiency with polyethylene glycolmodified adenosine deaminase. N Engl J Med 316:589-596
25. Culver KW, Anderson WF, Blaese RM 1991 Lymphocyte gene therapy. Human Gene Therapy 2:107 109

26. Bonthron DT, Markham AF, Ginsburg D, Orkin SH 1985 Identification of a point mutation in the adenosine deaminase gene responsible for immunodeficiency. J Clin Invest 76:894-897

27. Valerio D, Dekker BMM, Duyvesteyn MGC, van der Voorn L, Berkvens TM, van Ormondt H, van der Eb AJ 1986 One adenosine deaminase allele in a patient with severe combined immunodeficiency contains a point mutation abolishing enzyme activity. EMBO J 5:113 119

28. Akeson AL, Wiginton DA, States JC, Perme CM, Dusing MR, Hutton JJ 1987 Mutations in the human adenosine deaminase gene that affect protein structure and RNA splicing. Proc Natl Acad Sci USA 84:5947-5951

29. Akeson AL, Wiginton DA, Dusing MR, States JC, Hutton JJ 1988 Mutant human adenosine deaminase alleles and their expression by transfection into fibroblasts. J Biol Chem 263:16291-16296

30. Hirschhorn R, Chakravarti V, Puck J, Douglas SD 1991 Homozygosity for a newly identified missense mutation in a patient with very severe combined immunodeficiency disease due to adenosine deaminase deficiency. Am J Hum Genet 49:878-885

31. Hirschhorn R 1992 Identification of two new missense mutations (R156C and S291L) in two ADA-SCID patients unusual for response to therapy with partial exchange transfusions. Human Mutation 1:166-168

32. Hirschhorn R, Tzall S, Ellenbogen A, Orkin SH 1989 Identification of a point mutation resulting in a heat-labile adenosine deaminase (ADA) in two unrelated children with partial ADA deficiency. J Clin Invest 83:497-501

33. Hirschhorn R, Tzall S, Ellenbogen A, Eng F 1990 "Hot spot" mutations in adenosine deaminase (ADA) deficiency. Proc Natl Acad Sci USA 87:61716175

34. Markert ML, Hutton JJ, Wiginton DA, States JC, Kaufman RE 1988 Adenosine deaminase deficiency (ADA) due to deletion of the ADA gene promoter and first exon by homologous recombination between two Alu elements. J Clin Invest 81:1323-1327

35. Berkvens TM, van Ormondt H, Gerritsen EJA, Meera Khan P, van der Eb AJ 1990 Identical 3250-bp deletion between two AluI repeats in the ADA genes of unrelated ADA-SCID patients. Genomics 7:486-490

36. Cooper DN, Krawczak D 1990 The mutational spectrum of single base-pair substitutions causing human genetic disease: patterns and predictions. Hum Genet 85:55-74

37. Jenkins T, Lane AB, Nurse GT, Hopkinson DA 1979 Red cell adenosine deaminase (ADA) polymorphism in Southern Africa with special reference to ADA deficiency among the !Kung. Ann Hum Genet 42:425-433

38. Hirschhorn R, Ellenbogen A, Tzall S 1992 Five missense mutations at the adenosine deaminase locus (ADA) detected by altered restriction fragments and their frequency in ADA- patients with severe combined immunodeficiency. Am J Med Genet 49:201-207

39. Markert ML, Norby-Slycord C, Ward FE 1989 A high proportion of ADA point mutations associated with a specific alanine-to-valine substitution. Am J Hum Genet 45:354-361

40. Wilson DK, Rudolph FB, Quiocho FA 1991 Atomic structure of adenosine deaminase complexed with a transition-state analog: understanding catalysis and immunodeficiency mutations. Science 252:1278-1284

41. Chang ZY, Nygaard P, Chinault AC, Kellems RE 1991 Deduced amino acid sequence of Escherichia coli adenosine deaminase reveals evolutionarily conserved amino acid residues: implications of catalytic funtion. Biochemistry 30:2273-2280

42. Arredondo-Vega FX, Kurtzberg J, Chaffee S, Santisteban I, Reisner E, Povey MS, Hershfield MS 1990 Paradoxical expression of adenosine deaminase in $\mathrm{T}$ cells cultured from a patient with adenosine deaminase deficiency and combined immunodeficiency. J Clin Invest 86:444-452 